NISTIR 7965

\title{
A Methodology for Handling Standards Terminology for Sustainable Manufacturing
}

Anantha Narayanan

David Lechevalier

KC Morris

Sudarsan Rachuri

http://dx.doi.org/10.6028/NIST.IR.7965

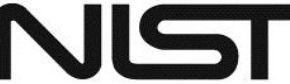

National Institute of Standards and Technology U.S. Department of Commerce 
NISTIR 7965

\section{A Methodology for Handling Standards Terminology for Sustainable Manufacturing}

Anantha Narayanan

David Lechevalier

KC Morris

Sudarsan Rachuri

Systems Integration Division

Engineering Laboratory

http://dx.doi.org/10.6028/NIST.IR.7965

October 2013

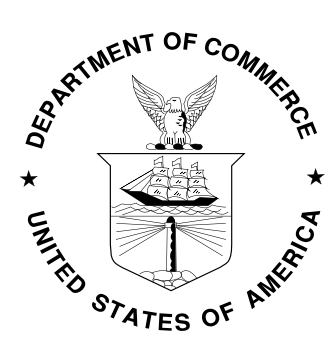

U.S. Department of Commerce

Cameron F. Kerry, Acting Secretary

National Institute of Standards and Technology

Patrick D. Gallagher, Under Secretary of Commerce for Standards and Technology and Director 


\section{Abstract}

To develop the discipline of sustainable manufacturing, the language of discourse needs to be properly and clearly communicated, for both manufacturers and consumers. As a result, a range of information standards that define the needed terminology is emerging. These standards span a wide range of technology, as well as geo-political areas, and serve multiple purposes. The task of understanding the challenges in achieving a sustainability objective is difficult when non-experts have to browse through several pages of document standards to find relevant information. Traditional document style standards and dictionary style definitions are limiting when it comes to getting a holistic picture of requirements imposed by the business objectives of sustainability. Our objective is to build a classification system for sustainable manufacturing terminology and a repository for knowledge dispersion to improve a reader's comprehension of this information and support decision-making. In this paper, we present an informatics approach to synthesize and classify the terminology defined in standards by studying the structure and relationships within standardized lexicons. 


\section{Table of Contents}

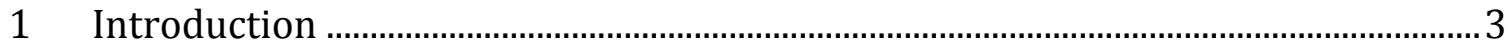

1.1 Informatics Approach ................................................................................

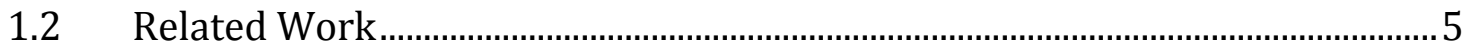

1.2.1 Standards for Sustainable Manufacturing .....................................................

1.2.2 Related work in taxonomies ...........................................................................

1.2.3 Knowledge organization ...............................................................................

1.2.4 Information Visualization ............................................................................

2 Knowledge Representation in NOVIS .....................................................................

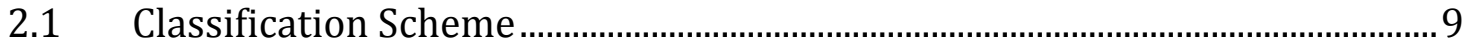

2.2 OWL Ontology ………………………………………………………….... 12

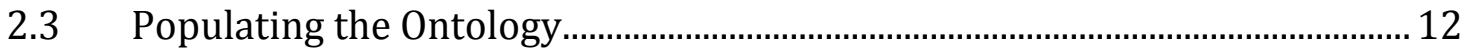

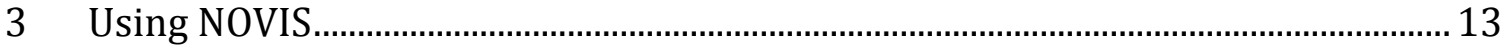

$3.1 \quad$ Visualization ........................................................................................... 14

3.2 Query Engine .................................................................................................. 16

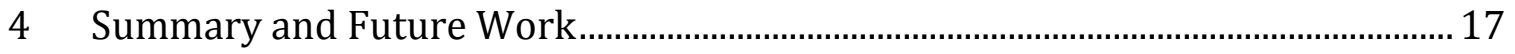

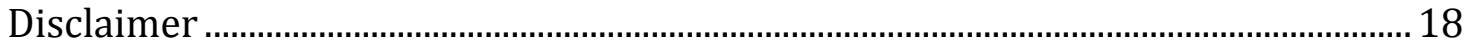

5 References ............................................................................................................ 18 


\section{Introduction}

Technology is evolving at a rapid rate today. As part of that evolution a network of information standards also evolves. Standards span a wide range of technology areas and serve multiple purposes. For instance, performance standards ensure that technology components perform as expected so that the components can be integrated into larger products and enterprises. Other standards define business processes to support interoperability between enterprises. Still more standards prescribe regulations that constrain how different materials may be used and limit the negative impacts of different processes and practices. This conglomerate of standards contributes towards an orderly yet rapid development of new technology.

One of the functions of these standards is the containment of technology such that a sustainable system will evolve. The sustainability of the system can be measured by the impact the system has on the overall environment in terms of the resources consumed and produced by the system. In the manufacturing area this evaluation is discussed as the study of sustainable manufacturing. To develop the discipline of sustainable manufacturing, a language of discourse needs to be properly and clearly communicated, for both manufacturers and consumers. Producers need to clearly understand and articulate their requirements and the services they provide, while consumers need to understand the meanings of that information to make informed choices. As such a lexicon of sustainable manufacturing is evolving and is often captured in standards documents.

The rapidity of growth in this lexicon is further complicated through the globalization of commerce. The terms being used to discuss sustainable manufacturing are emerging in the form of research, policy, and regulations in locales all over the globe. Many of these are being formalized within standards that are also dispersed and disconnected. Concurrently, businesses are developing and producing products that will be marketed all over the globe. Businesses need to understand how their products relate to the concepts being promoted locally as sustainable manufacturing and at the same time have a global understanding of the sustainability of their practices.

In this paper, we present an informatics approach to synthesize and classify the terminology defined in standards by studying the structure and relationships within those lexicons. Traditional document style standards and dictionary style definitions are limiting when it comes to getting a holistic picture of sustainability requirements. The task of understanding the challenges in achieving a sustainability objective is difficult when non-experts have to browse through several pages of document standards to find relevant information. Recently, many standards have been made available as electronic documents, searchable on the web. However, these searches are usually simple text searches, with no direct relevance to the semantic content of the standards. Our objective is to build a classification system of sustainable manufacturing terminology and a repository for knowledge dispersion 
to assist in sustainability decision making (for both manufacturers and consumers). Therefore, not only do we collect the relevant sustainable manufacturing terminology, we also define a means for classifying these terms and constructing relationships between them. This structure will aid in improved understanding and decision- making ability.

\subsection{Informatics Approach}

Understanding the challenges of sustainable manufacturing can be a daunting task. While there are a diverse range of standards that address various aspects of sustainable manufacturing, it is very challenging for non-experts to navigate through the standards documents to identify information, and devise a plan of action, to address their sustainability objectives. Our approach to this problem is to organize the standards information in such a way that different standards can be related while still maintaining the connectivity back to their authoritative sources. The terminology of the standards is synthesized and classified based on a multifaceted classification scheme. We develop an ontological schema to capture the main concepts in sustainable manufacturing, and constructed an ontology that classifies a wide range of sustainable manufacturing terminology. We take a standards-centric approach in capturing a set of standard terminology that address the most critical aspects of sustainable manufacturing. Finally, we produce a prototype implementation to demonstrate a visual interface built upon the underlying logical framework. The work is designed in such a way that contributions may be solicited from experts to expand the network of information by including a wide range of standards. We expect that this approach will be relevant to other areas of study beyond sustainable manufacturing.

Figure 1 depicts the architecture of our system, the NIST Ontological Visualization Interface for Standards (NOVIS) ${ }^{1}$. NOVIS uses the Web Ontology Language (OWL) [1] for organizing terminology related to sustainable manufacturing. We develop a classification scheme for terminology related to sustainable manufacturing, by surveying the literature. We create an OWL representation of this classification scheme to be used by the NOVIS tool. Next, we extract terminology from a number of standards documents, and classify them according to the classification scheme. The collected terminology is represented as an OWL ontology, the technical details of which are described in Section 2. A query and control engine, and a visual interface are designed to present the information contained in the OWL ontology in an intuitive way to the user. In our future development, it will be possible to use the query and control engine to interactively modify and update both the classification scheme and the OWL ontology. These aspects are described in detail in Section 3. We use existing software tools that process OWL to produce a prototype implementation of this approach.

\footnotetext{
${ }^{1}$ NOVIS is a software tool developed at the National Institute of Standards and Technology (NIST) to provide an interactive visual interface to the terminology used in a variety of standards related to sustainable manufacturing [21].
} 


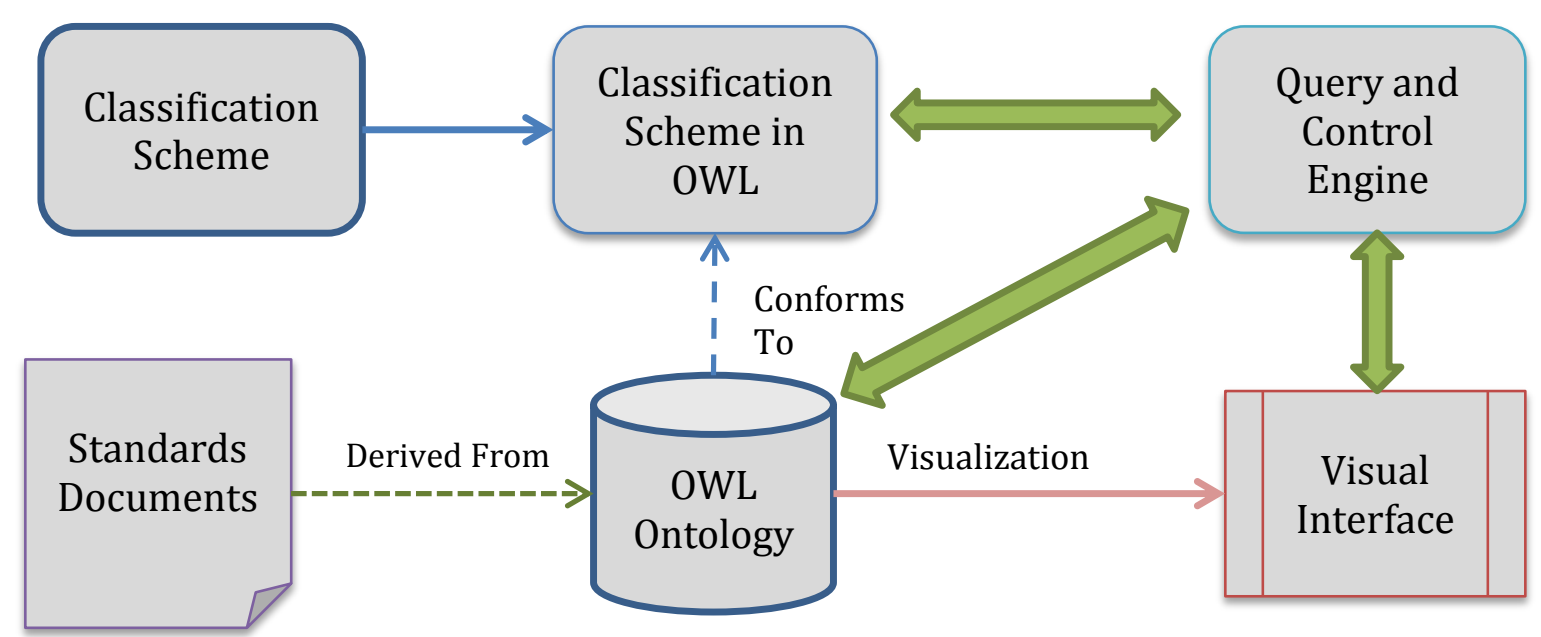

Figure 1: Architecture for NIST's Ontological Visualization Interface for Standards (NOVIS)

The remainder of this section provides background on the multiple facets of the work related to the project including various sustainability-related standards and work on knowledge representation and visualization. Section 2 goes into the details of the classification schema for sustainable manufacturing and the OWL implementation. Section 3 describes the query and visualization approach.

\subsection{Related Work}

The existing work relevant to our project can be divided into two distinct categories:

- sustainable manufacturing standards

- knowledge representation technology

The standards related to sustainable manufacturing are the driving force in undertaking this project. Over the last several years numerous standards have emerged in this area. These standards often overlap and yet are different both in form and in where they apply. The text in these standards can often be difficult to interpret in different contexts. Different words might be used for the same meaning, while the same words might also be used but with somewhat different meanings in different contexts. Furthermore, the standards can apply across different geo-political regions or in different industries. For an end-user, understanding these differences and keeping track of the implications for their business can be overwhelming.

Secondly, work on knowledge representation and the technology, which supports it, is also an evolving field. We propose a new way of defining terminology in standards that makes full use of the emerging technology in this area. This approach enables intelligent navigation and advanced querying. Intelligent navigation uses colors and visual cues to navigate to related concepts as captured in the ontology. We are experimenting with applying recent advances in the technology for graph visualization and visual dictionaries. Advanced querying enables queries to be constructed at varying levels of abstraction. For example we 
can find all "related resources," or narrow down the search to specific types of resources. In this case, we are applying ontology technology to the definition of a lexicon for sustainable manufacturing.

\subsubsection{Standards for Sustainable Manufacturing}

Standards are defined as "the common and repeated use of rules, conditions, guidelines or characteristics for products or related processes and production methods, and related management systems practices." [2] Standards play a crucial role in manufacturing, serving as best or recommended practices that manufacturers should follow to produce quality products. Regulations are used to specify mandatory requirements that must be met under specific laws, and implement general agency objectives. Many governments have enforced regulations that restrict the manufacture of products that pose a threat to our environment. For example, the Restriction of Hazardous Substances (RoHS) directive [3] is a European regulation that restricts the use of certain harmful chemical substances in electronic products, and is enforced in several countries. Regulations are often supported by voluntary standards that may serve as a basis for rule making or as general guidelines for compliance. For instance, IPC-1752 [4] is a material declaration standard that can be used to declare compliance with the RoHS regulation. Standards and regulations play an important role in sustainable manufacturing due to the complex nature of cross-domain concerns it involves.

In recent times, we have seen an increasing number of standards and directives related to sustainability. Previous work at NIST focused on characterizing and cataloging a number of these standards [5]. This work highlighted the importance of taking an informatics approach to resolve the complexities and ambiguities in these standards. One of the important issues was the use of terminology in different and sometimes conflicting ways. Moreover, information about these standards is usually provided by disparate bodies and often does not consider wide ranging impacts and relations to other bodies of work. In a workshop on sustainable manufacturing conducted at NIST in 2009 [6], industry participants identified access to and understanding of information as the main hurdles in incorporating sustainable practices in their businesses. One of the requirements identified was the need for a consolidated repository of information that takes a holistic view of sustainability standards, and allows users to navigate through various related standards along clearly defined logical and physical relationships.

\subsubsection{Related work in taxonomies}

The classification scheme we have adopted in this paper relies heavily on certain existing taxonomies. The Manufacturing Processes Reference Guide [7] provides a taxonomy of manufacturing processes. This taxonomy is based on the Fabrication Process Taxonomy [8] and organizes processes in a tree taxonomy based on their impact on the materials that are processed. Figure 2 shows a portion of this taxonomy. Xue et al. [9] recommend using such a process classification system to understand the nature of the process and make environmentally friendly choices. 
Our work takes advantage of these existing taxonomies to provide a classification scheme for sustainable manufacturing.

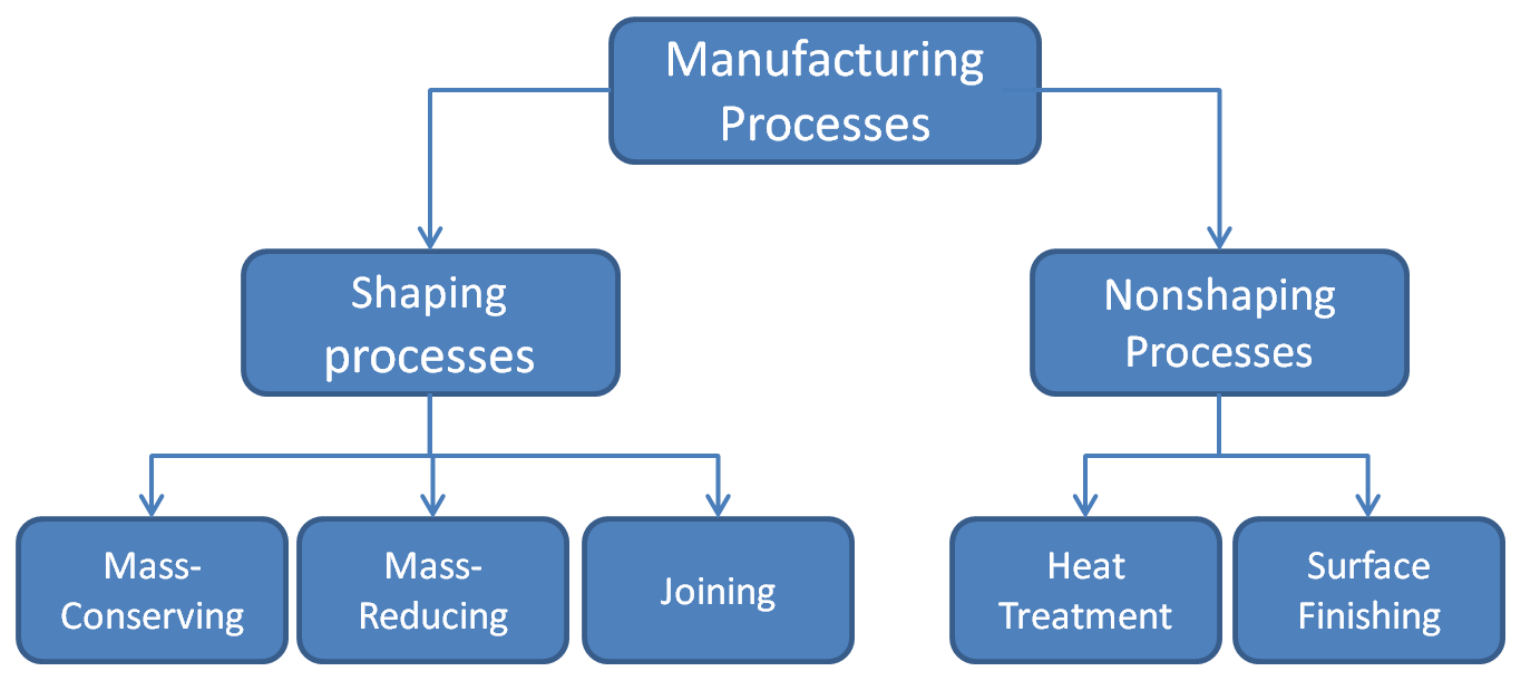

Figure 2: Portion of manufacturing process taxonomy [7]

Snomed CT [10] is a taxonomy related to health care. It resulted from the merging of two terminologies and contains more than 311,000 concepts. This taxonomy is enriched by relationships that add a semantic meaning to the hierarchical organization. This taxonomy provides standardized definitions for medical terminology. The electronic health record system is based on this classification. We propose a similar approach to semantically enrich existing taxonomies related to manufacturing, to provide a classification scheme for sustainable manufacturing standards.

\subsubsection{Knowledge organization}

The knowledge organization technology that has influenced our work is, first, that of ontologies to represent information and, secondly, work in visual dictionaries for presenting information.

Ontologies are used in several domains, like biology, health care, and even natural language processing, to create classification schemes for these domains. Perhaps the first formal classification schemes were taxonomies. Taxonomy was originally the discipline of classification of living organisms in bacteriology, botany and zoology. However, its use has now spread to other sciences and the term taxonomy is used to describe systems or hierarchical classification methods for inventorying objects, concepts, information of a given area to:

- $\quad$ determine the behavior to adopt towards a given object

- $\quad$ predict the behavior of an object

- $\quad$ understand a phenomenon to be able to then act

Where taxonomy is a tree-based classification scheme, other schemes may form networks of classification. Trees are useful for decomposing a single domain into mutually exclusive classes; however, in a multidisciplinary world, such as that 
covered by sustainable manufacturing, classification networks are useful for crossing domains. Our work proposes a set of taxonomies in different disciplines, such as standards documentation, and relates these through classifications which span the taxonomies. This organization is suitable for our goal of supporting comprehension and retrieval of information concerning sustainable manufacturing. In contrast the traditional form of representing terminology in standards is dictionary based. In a dictionary, it is difficult to trace relationships between terms and other concepts. For instance, a dictionary definition does not explicitly capture relationships between a term and other terms, other standards, or sustainable manufacturing concepts. By building a classification scheme, we explicitly establish these relationships not only as links to other concepts but also as relationships with a semantic meaning. Capturing semantic relationships presents great possibilities, such as context-driven presentation of information and advanced querying capabilities. Such a semantic network can be implemented using an ontology.

To facilitate search, an ontology is used as a structuring device for an information repository (e.g., documents, web pages, names of experts); this supports the organization and classification of repositories of information at a higher level of abstraction than is commonly used today [11]. An ontology is a semantic network that allows a user to follow a stream of thought through querying and navigation, as opposed to a loosely related list of search engine results [12].

Perhaps the most widely adopted language for representing ontologies today is OWL. OWL was developed for connecting disparate information across the Internet, i.e., the World Wide Web. It is suitable for connecting information contained in a wide variety of formats, in dispersed locations, and organized as loose and flexible networks. OWL ontologies are based on a set of axioms, which define classes and relationships in order to contain the data. OWL is designed for being semantically interpretable by computer applications, and not primarily for human readability.

A recent effort from the World Wide Web Consortium (W3C) into what is known as Simple Knowledge Organization System (SKOS) [13] is a more specific set of standards for representing structured controlled vocabularies. SKOS is built upon the Resource Description Framework (RDF) [23]. It may be used on its own, or in combination with more-formal languages such as OWL. There are many software tools that support the OWL format and more are emerging that are specialized for SKOS. Protégé [14] is an open-source application for creating, visualizing, and manipulating OWL ontologies. Additional plugins may be developed for Protégé for specific tasks. In particular, a SKOS Editor developed by the University of Manchester makes it possible to create and manage SKOS vocabularies [24]. SPARQL [15] is a query language used to retrieve and manipulate data in RDF and OWL. When using SKOS with OWL, we may use SPARQL to execute queries on the ontology. Based on these queries it is possible to extract specific information that is contained in the ontology. 


\subsubsection{Information Visualization}

Dynamic visualization tools are useful to help users quickly understand and evaluate the data. For navigating, information visualization technology [16] has been developed to interactively show concepts. In this context, information visualization technology is a means by which concepts or data are graphically represented to facilitate comprehension. The technology is becoming increasingly popular in our net-centric world. One example of such technology is the graphical dictionary Visuwords (http://www.visuwords.com/) based on Wordnet, a large lexical database of English [17]. Another example is DebateGraph [18], which is a cloud-based service that offers individuals and communities a way to learn about and deliberate and decide on complex issues. According to Nicholas H. Lurie \& Charlotte H. Mason in 2007 [19], there was no doubt that visual representations will become more prevalent for decision making. For instance, business intelligence is a set of theories, methodologies, processes, architectures, and technologies that transform raw data into meaningful and useful information often displayed as graphs.

\section{Knowledge Representation in NOVIS}

In this section we describe the NOVIS classification scheme, how we turn that into an OWL ontology, and finally how that ontology is populated with the contents of various standards.

\subsection{Classification Scheme}

Our approach to building the classification scheme is to define taxonomies for each of the various categories of concepts related to sustainable manufacturing, and to capture relationships of the concepts between these taxonomies in a schema or package. The terminology used in sustainable manufacturing is classified accordingly. This separation allows for concurrent and distributed development of the system. The taxonomies in the classification scheme are shown in Figure 4. They are arranged in a hierarchy. We use a combination of abstract concepts and concrete concepts to facilitate the task of capturing relationships between the different facets of the classification scheme. The Core package is unique in that it defines abstract constructs for classification, which are used by and refined in the other packages. For example, it defines the abstract concept Classifier, which allows the concepts in the taxonomies to be specialized in similar ways between the other packages. 


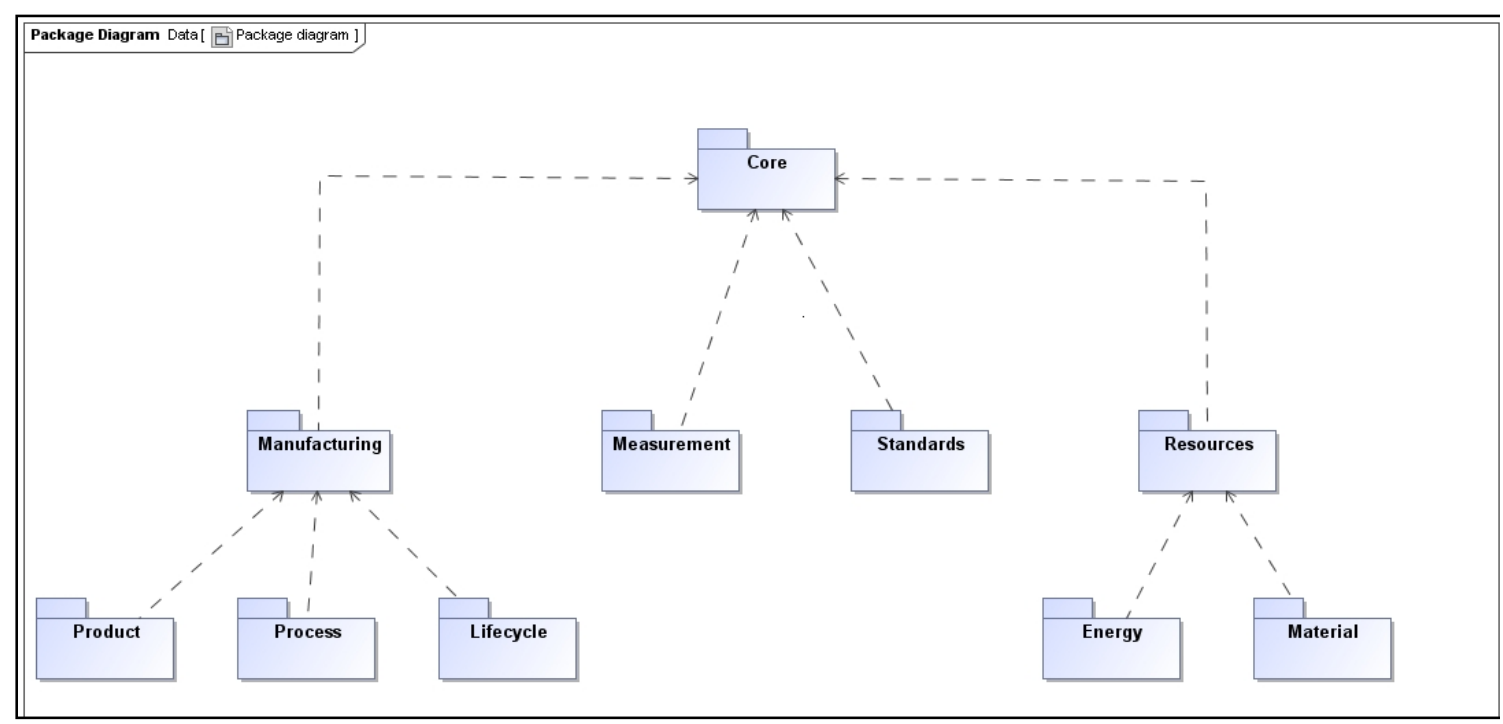

Figure 4: Overview of Packages in the Schema

The Standards package defines concepts such as Standard and Term. Using this scheme, we can define low level relationships between the concrete concepts Standard and Term, or define a more abstract relationship between the concept Term and any other concept which is a type of abstract concept called Classifier. As we will see later, this will allow us to capture a complex network of information and construct queries to find information easily. Besides the abstract Core package, we have identified four main packages: Manufacturing, Measurement, Standards,

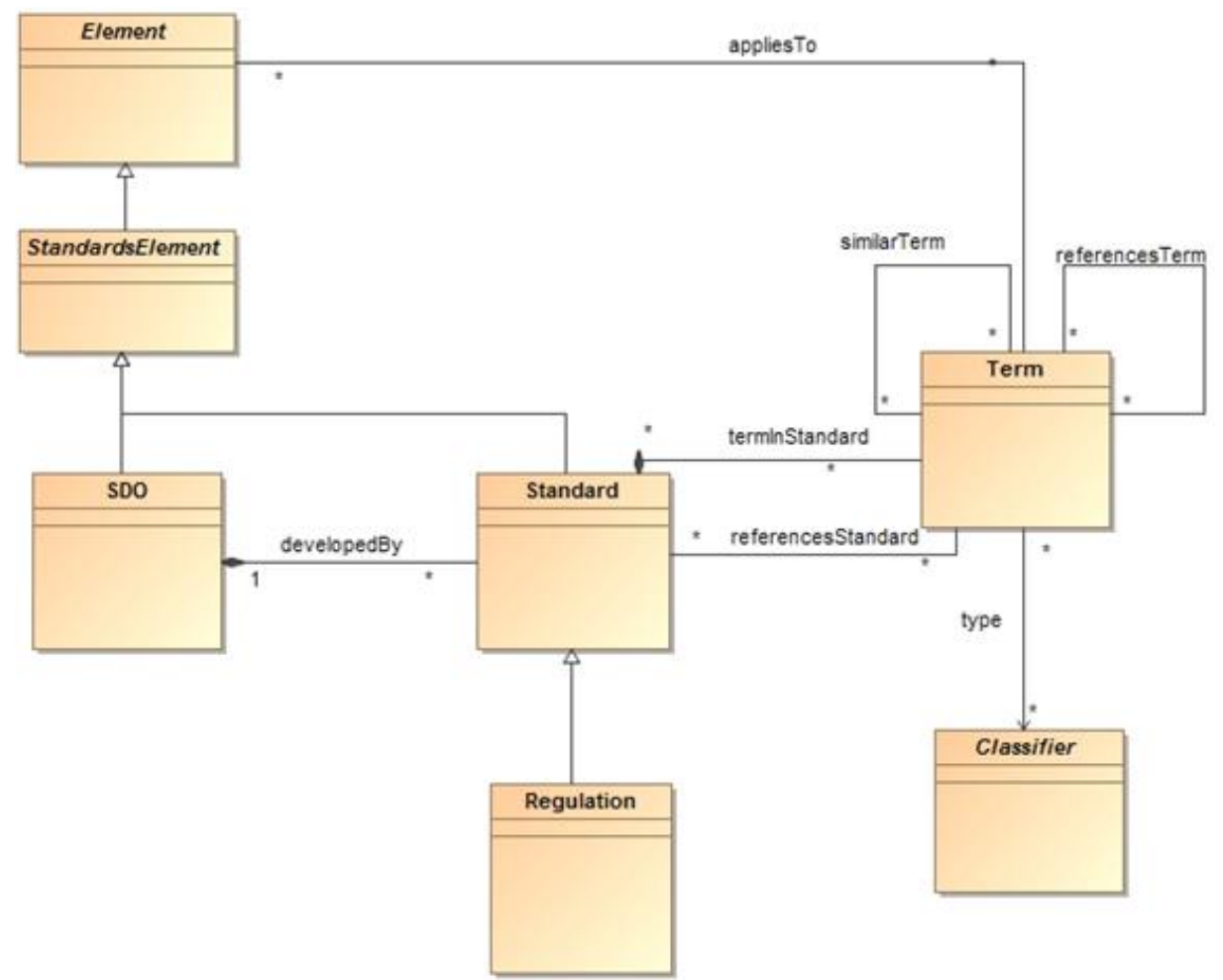

Figure 3: Overview of Standards Package 
and Resources packages. The Manufacturing package covers concepts related to manufacturing including process types, product categories, and product life cycle. The Measurement package focuses on the evaluation of physical quantities related to the sustainable manufacturing. The Standards package contains concepts such as Standard and Term, which classify standards and terminology in sustainable manufacturing. The Resources package covers the material used in the sustainable manufacturing. These may be further divided into sub-packages. This arrangement allows the individual packages to be developed by domain experts, and integrates them at a higher level. In the rest of this section, we will focus on the Standards package, since our initial goal is to extract, organize, and store terminology contained in standards documents. Other packages will be organized in a similar fashion.

The Standards package is shown in Figure 3. In this package, we first define the abstract concept called StandardsElement as a subtype of the abstract concept Element from the Core package. We define the concrete concepts SDO, Standard, Term etc. as subtypes of the abstract concept StandardsElement. The concept SDO represents standards development organizations. The concept Standard is used to represent individual standards, and the concept Term is used to represent terminology from standards (terms may be single words or phrases). We have also defined the following relationships between these concepts. A Standard can contain several Terms. This is captured by the relationship termInStandard between the two concepts. A Term can be related to other Terms using relationships such as referencesTerm or similarTerm, and also with other elements of our schema using relationships such as appliesTo.

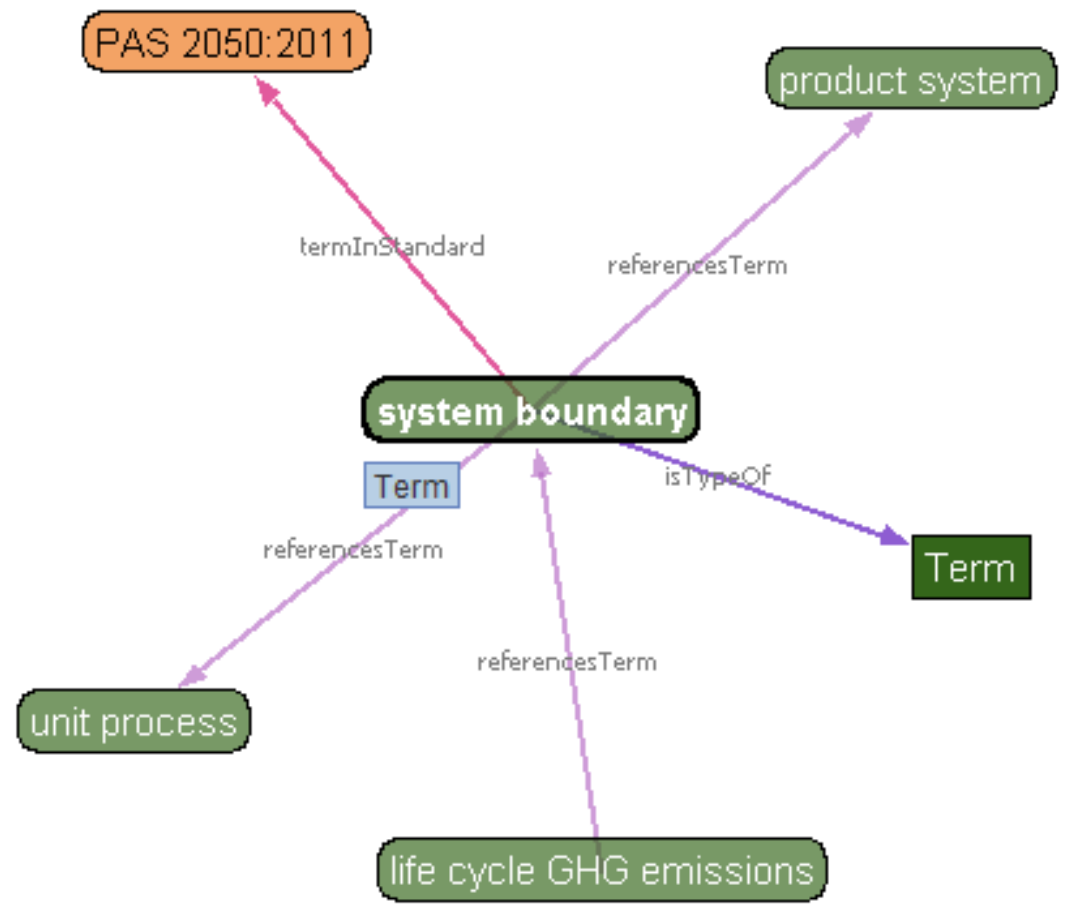

Figure 5: System boundary term and its relationships 
For instance, consider the term system boundary from the Standard PAS2050:2011 [20]. We can symbolize this connection between the term and the standard with the composition relationship named termInStandard. Furthermore, in its definition, the term contains several other terms like unit process. We can symbolize this connection with the relationship named referencesTerm. Figure 5 illustrates this example. As you can see, system boundary is also referenced by the term life cycle GHG emissions. Moreover, since the schema defines the appliesTo relationship between Term and the abstract class Element, you can relate any Term to a specialized Element from another package. As mentioned above, we have surveyed several standards developed by different SDOs (Standards Development Organizations) to extract the relationships and classes defined in the Standards package.

\subsection{OWL Ontology}

The taxonomies described in the previous section are represented as an OWL ontology for use in the NOVIS application. The following OWL mechanisms are used to represent the classification scheme as an OWL ontology:

- Classes correspond to the classes identified in the taxonomies. The classes are all rooted in the "Thing" class which is fundamental to OWL. We do not make a distinction between abstract and concrete concepts in OWL, due to technical differences in the way OWL concepts are treated ${ }^{2}$.

- Data properties are used to capture the attributes or properties of the concepts.

- Object properties are used to capture relationships between concepts.

Once we have created the OWL ontology representing the classification scheme, we can populate this ontology with terminology extracted from standards documents, by creating OWL individuals of the appropriate classes.

\subsection{Populating the Ontology}

Once the schema has been encoded in OWL, we add terminology from standards to populate the ontology. We do this by manually extracting terms from a standard, and classifying the terms based on the schema. Initially, this is a tedious and timeconsuming task that requires domain expertise and a good understanding of the classification scheme. However, we are developing tools and techniques to simplify and automate this task. For instance, we have developed a plug-in for the Protégé tool that reads terms and definitions from a Microsoft Excel file and imports them into the OWL ontology.

Terms extracted from standards documents are encoded as OWL individuals of the class Term. The standard's definition of the term is entered as the value of the definition data property for the corresponding OWL individual. We investigate the

\footnotetext{
${ }^{2}$ In OWL, the relation between classes and the instances they represent is purely logical. An 'instance' of a class in OWL is also considered an instance of all of the super-classes of that class.
} 
definition to find other related terms and standards, and establish these relations in the ontology.

As an example, consider the PAS2050 standard. We create an OWL individual of the class Standard for this standard, and set its name and description properties accordingly. We then manually add the terms from PAS2050 to our ontology. For example, PAS2050 defines the term "anticipated life cycle greenhouse gas emissions". We create an OWL individual in our ontology to represent this term. We transcribe the name and definition as data properties of this individual. In OWL the term is related to other terms through object properties. For instance, "anticipated life cycle greenhouse gas emissions" is related to the PAS2050 standard through the object property termInStandard. The value of this property is set to be the OWL individual named "PAS2050". Moreover, the term contains other terms in its definition. In the case of this definition, some terms are explicitly called out through the use of the standard's own numbering system but that is not always the case. These relationships are also captured through object properties but are distinguished by the use of different types of object properties. In this case the object property named referencesTerm is used to relate the terms. Similarly, the "anticipated life cycle greenhouse gas emissions" term is related to the other terms by using this object property. In this way, the schema allows us to capture various terms as well as relationships between terms.

Capturing the terminology as a network of information in this manner allows one to develop advanced user interfaces for the information. The network can be displayed as a visual graph to aid comprehension of the information. Also, advanced queries can be written based on the relationships represented, thereby facilitating the use of the standard. The following section describes the NOVIS tools that demonstrate this approach.

\section{Using NOVIS}

Based on the ontology described in the previous section, we have developed two ways to access the information: an interactive visual interface for viewing the network of terminology and a query interface for locating information. As a large graph or network of information, we make use of established methods of viewing and navigating graph networks to visualize the ontology. We have also developed a query interface for generating advanced queries by creating a set of predicates to describe the concepts and relations defined in the ontology. Here we leverage SPARQL [15], a query language applicable to OWL, though the user does not require any knowledge of SPARQL to use the advanced query interface. Note that the NOVIS interface is designed to operate independently from a given ontology. In other words, any OWL ontology meeting certain requirements can be loaded into the tool as is explained in [21]. This separates the interface concerns from those of the particular ontology being used and allows the ontology and interface to evolve 
separately. This feature is important as new discoveries are constantly being made in the area of sustainable manufacturing, causing the standards and other information in this area to grow and evolve rapidly.

\subsection{Visualization}

Visualization in NOVIS is designed to be interactive and intuitive and is guided by the classes and relationships that compose the ontology. We have used the open source API called Prefuse [22] to build our ontology visualization tool. This toolkit allows us to quickly create customized representations of the different types of classes and relationships in the ontology and present them in a graphical form. The classes and individuals from the ontology are represented as nodes in the graph. To distinguish them, the classes are represented by rectangles, and individuals are represented by rounded rectangles. To distinguish the classes themselves each is represented as a different color. Arrows symbolize the relationships in the ontology, which have the underlying representation of object properties between the individuals. The direction of the arrow corresponds to the ownership of the relatio nship. Data properties are not included in the graphical representation but are displayed in text format as is shown in Figure 6. Each element in the interface, whether graphical or text-based, is interactive.

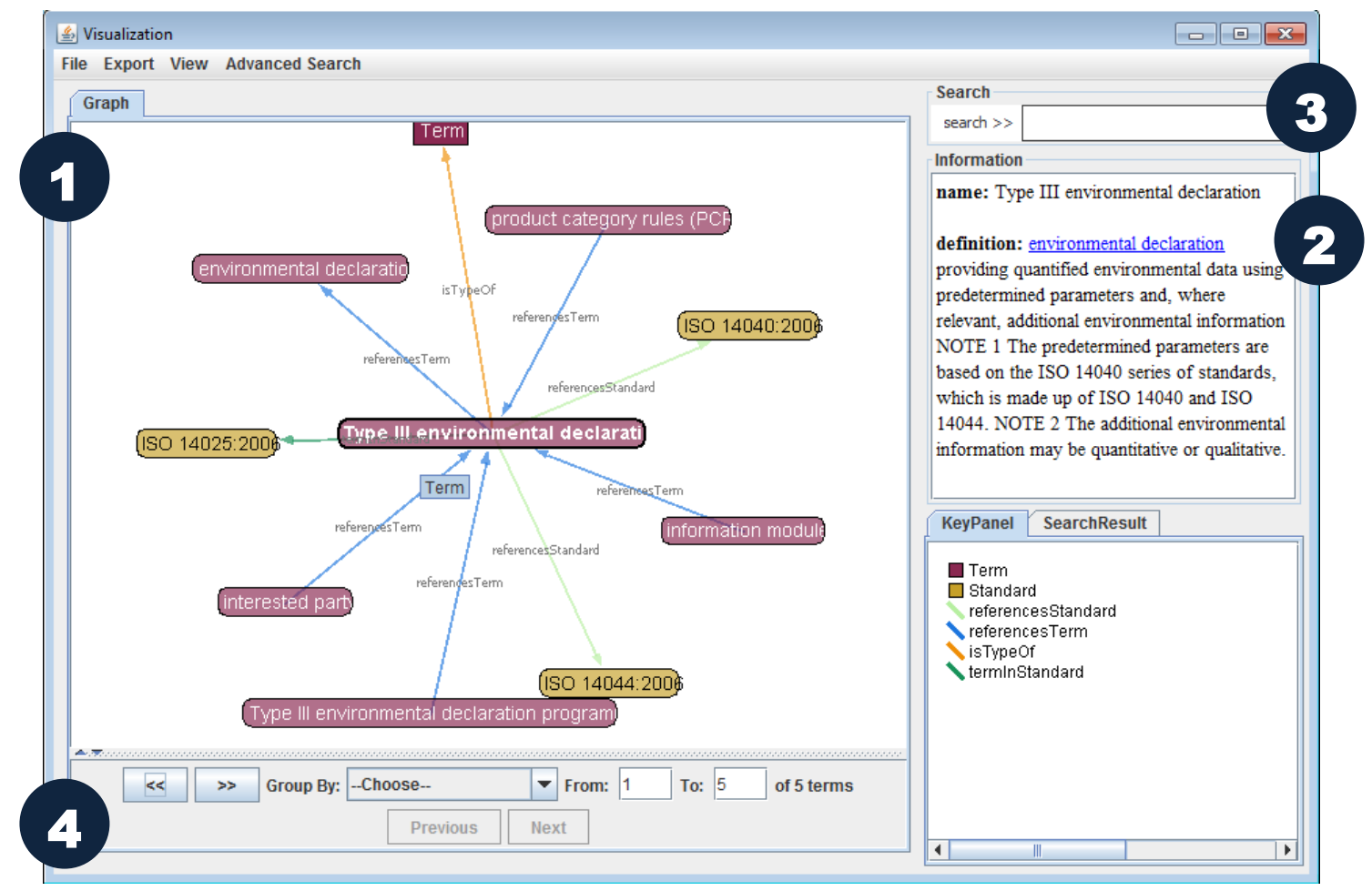

Figure 6: NOVIS Visualization Tool 
The interface as shown in Figure 6 consists of the four parts described below:

1. Graphical View: The box on the left shows a graphical view of the selected term, and its immediate neighborhood of related terms, standards and other elements. This is a clickable interactive interface. The terms are presented as a graph where the nodes represent individual terms, standards and other concepts, and edges represent relationships between these concepts. Colors and shapes are used to aid in understanding the types of these concepts. This visual representation provides an intuitive overview of the term and its immediate context. Clicking on a node in this view redraws the graph by opening the clicked node and displaying its immediate neighborhood of related nodes. This allows the user to navigate through the large and dense underlying network of information by viewing a small portion of it at a time, and traversing relations that are of interest.

2. Descriptive Information: The information panel on the right shows descriptive information about the selected item. For example, when a term is selected in the graph on the left, the information panel gives information about that term, such as its definition and source. The definition text itself is hyperlinked, so that clicking on certain phrases in the definition will lead the user to other terms that will help him/her better understand the terminology.

3. Quick Search: The quick search box on the right of the tool window provides a simple string search, which matches partial strings in the ontology to give a quick list of results. Note this is much more basic than the advanced search that is described in the following section.

4. Navigation Panel: The navigation panel at the bottom assists in navigating the information network. Forward and back buttons are provided for simple navigation through terms viewed so far. More advanced options include the ability to group the terms based on certain properties, such as a term's relationship to specific standards, to assist in more directed navigation of the information network.

The navigation panel and other aspects of the interface are described in more detail in [21]. One feature of the navigation panel that is especially useful for comprehension is the ability to filter based on values for different types of relationships. This feature is accessible through the "Group By" selection in the navigation panel. With Group By a user choses the type of relationship of interest and then selects a value for that relationship. For example to view only those terms within a particular standard, one would chose the termInStandard relationship and provide the value for the standard of interest, perhaps PAS2050 from the example above. The interface then highlights anything in the graph that is contained in that standard. 


\subsection{Query Engine}

The tool supports an advanced query interface, which allows the user to specify search predicates based on the schema. The SPARQL language is used for querying the ontology. We have designed this interface to allow users without any knowledge of OWL or SPARQL to define the predicates, by directly referring to the concepts and relationships defined in the schema. Figure 7 shows the query interface window. Each row at the top of the window is a predicate. The user can add or remove rows to customize their query. The Qualified name column identifies the concept or relation in the schema that the user is interested in, and plays a crucial role in forming the query. To facilitate non-experts in generating specific queries, the dropdown lists in this column provide ready access to the concepts and relationships defined in the schema. For example, the user can search for a specific type of element, such as Term, and constrain the search by specifying additional constraints on the properties of the matched individuals. The middle box in the figure is the OWL query in SPARQL which is automatically generated from the predicates. The list at the bottom of the window contains the search results. Clicking on an item in this result list shows that item in the visualization window, along with its

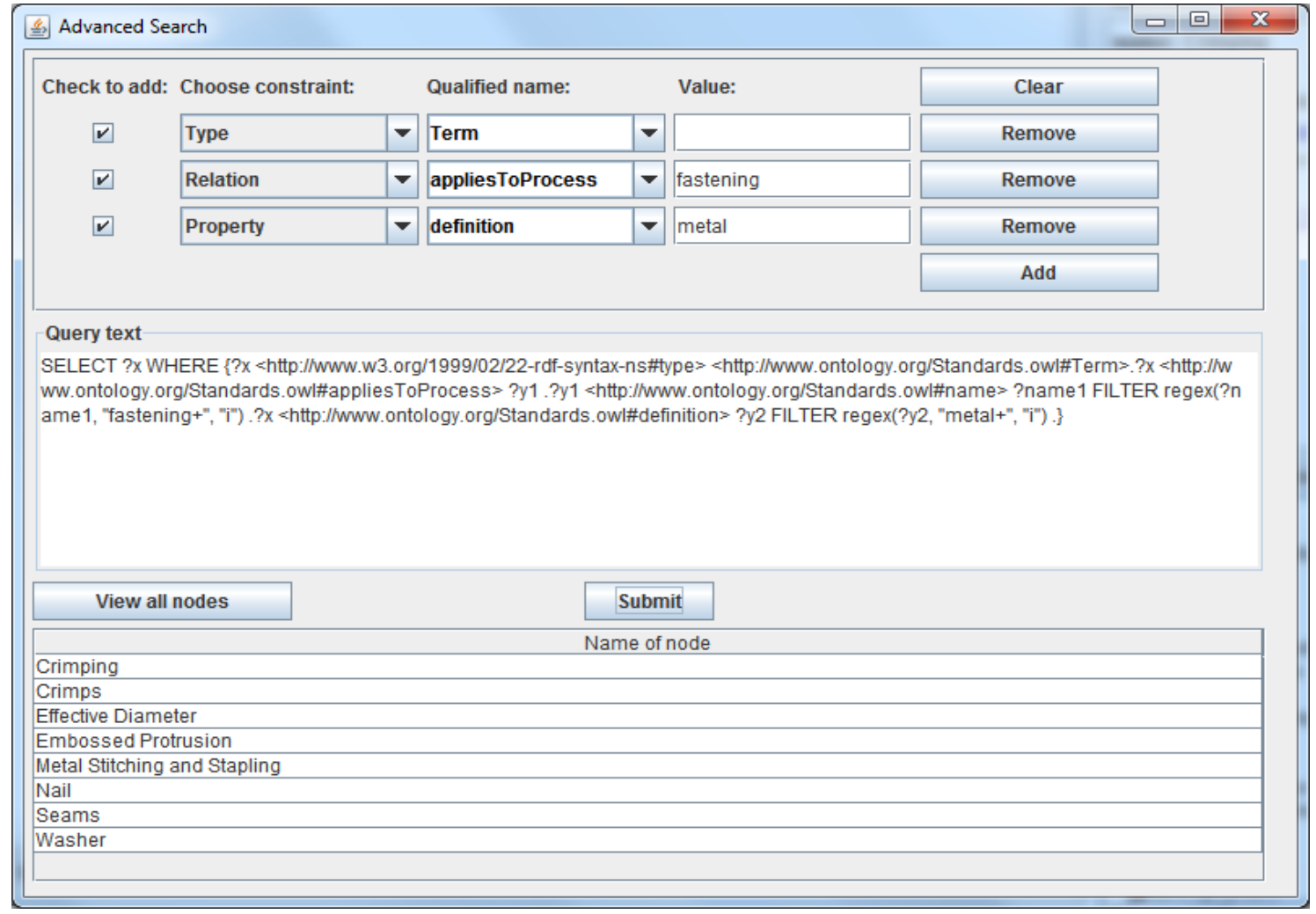

Figure 7: Advanced Query Interface

description and immediate relationships to other elements.

The query in Figure 7 is designed in the following way. The user wishes to find terms that are related to the metal fastening operations. The user generates a query by creating the following predicates: 1 ) match a term by finding individuals of type 
Term; 2) match only those terms that apply to the 'fastening' process using the relation appliesToProcess; and 3) match only those terms that also have the word 'metal' in their definition. Executing this query presents the relevant terms, and leads the user to other relevant standards and sustainability concepts by following the relationships in the visualization. This kind of information discovery is far more difficult using traditional document-based terminology repositories.

Note that to use the advanced query interface, the user must be familiar with the schema, even though no detailed knowledge of OWL or SPARQL is required. We believe that a well-constructed ontology will be easy to understand and use with very limited training. To assist the user in making the best use of the query interface, the tool provides easy access to the schema elements as drop-down lists, and also a tool tip describing the meaning of the chosen element.

\section{Summary and Future Work}

Sustainable manufacturing is a rapidly developing area. An understanding of the global necessity for sustainability has led to rapid development of new standards to aid sustainable manufacturing. An important part of correctly understanding and interpreting these standards lies in the correct interpretation of the terminology in standards. In this paper, we have shown an informatics approach to communicating standards terminology by categorizing them using a well-defined schema, and providing an intuitive visual navigation mechanism to learn about them. An advanced querying interface allows users to find terminology- and standardsrelated information easily. This framework allows manufacturers to find crucial standards-related information, without having to learn the tedious details of how standards documents must be read and interpreted.

In our future work, we will enhance the schema to cover the wide range of areas that impact sustainable manufacturing. We will also survey more standards documents and to create an OWL repository of standards that can be navigated visually and queried upon using our framework.

While the main goal of this work is to improve user comprehension of standards related to sustainable manufacturing, we also believe that this framework can aid the development of the standards themselves. One of the most painstaking tasks in the development of standards is the collection and consolidation of terminology to be used in the standard. The development and implementation of standards can be greatly accelerated if it is easier to manage the terminology in standards. Our future work will investigate the application of our framework for standards development. Our framework can facilitate the reuse of terminology from other standards, and in keeping track of changes to definitions of terms in related standards. The maintenance of standards can also be improved by applying our framework to record, store and maintain standards information. 


\section{Disclaimer}

Certain commercial software products or services may be identified in this paper. These products or services were used only for demonstration purposes. This use does not imply approval or endorsement by NIST, nor does it imply that these products are necessarily the best for the purpose.

\section{References}

1. W3C OWL Web Ontology Language Reference; Available from: http://www.w3.org/TR/owl-ref/

2. National Institute of Standards and Technology. What are Standards? 2012; Available from: http://nist.gov/standardsgov/definestandards.cfm

3. Directive Restriction of Hazardous Substances, Directive 2002/95/EC of the European Parliament and of the Council of 27 January 2003 on the restriction of the use of certain hazardous substances in electrical and electronic equipment, in Official Journal of the European Union, p. 19-23, 2003.

4. IPC-1752A. IPC-1752A - Materials Declaration Management; Available from: www.ipc.org

5. National Institute of Standards and Technology. Sustainability Standards Portal, 2011; Available from: http://www.mel.nist.gov/msid/SSP/

6. Rachuri, S., et al., Sustainable Manufacturing: Metrics, Standards, and Infrastructure - Workshop Report, NIST Interagency/Internal Report (NISTIR) - 7683, 2010; Available from: http://www.nist.gov/manuscript-publicationsearch.cfm?pub_id=905065

7. Todd, R.H., D.K. Allen, and L. Alting, Manufacturing processes reference guide. Industrial Press Inc., ISBN: 978-0831102005, 1994.

8. Dell, K. A., Fabrication Process Taxonomy, Monograph (Brigham Young University. Computer Aided Manufacturing Laboratory), 1980.

9. Xue, H., et al., Manufacturing process classification system for improved environmental performance, in SAE World Congress, Michigan, USA, 2000.

10. Stearns, M.Q., et al., SNOMED clinical terms: overview of the development process and project status, Proceedings of the American Medical Informatics Association (AMIA) Symposium, 2001.

11. Uschold, M. and M. Gruninger, Ontologies and semantics for seamless connectivity, ACM SIGMod Record, 33(4): p. 58-64, 2004.

12. Bhat, T.N., Semantic Web for Chemical Genomics-need, how to, and hurdles, WMSCI 2006: 10th World Multi-Conference on Systemics, Cybernetics and Informatics, Volume VII, Proceedings 1-6, 2006.

13. W3C, SKOS Simple Knowledge Organization System - Home Page. 2009; Available from: http://www.w3.org/2004/02/skos/

14. Protégé, Stanford Center for Biomedical Informatics Research, 2013; Available from: http://protege.stanford.edu/

15. SPARQL Working Group. SPARQL W3C Recommendations, 2012; Available from: http://www.w3.org/2009/sparql/wiki/Main Page 
16. Ware, C., Information visualization: perception for design, Morgan Kaufmann, ISBN: 978-1558608191, 2012.

17. Miller, G.A., WordNet: a lexical database for English, Communications of the ACM, 38(11): p. 39-41, 1995.

18. Baldwin, P. and D. Price, Debategraph, 2008; Available from: http://debategraph.org/home

19. Lurie, N.H. and C.H. Mason, Visual representation: Implications for decision making, Journal of Marketing, p. 160-177, 2007.

20. PAS2050, Specification for the assessment of the life cycle greenhouse gas emissions of goods and services, British Standards Institution, 2011.

21. Lechevalier, D., et al., NIST Ontological Visualization Interface for Standards User's Guide, NIST Interagency/Internal Report (NISTIR) - 7945, 2013;

Available from:http://www.nist.gov/manuscript-publicationsearch.cfm?pub id=913851

22. Heer, J., S.K. Card, and J.A. Landay. Prefuse: a toolkit for interactive information visualization, In Proceedings of the SIGCHI conference on Human factors in computing systems, pp. 421-430, ACM, 2005.

23. W3C, Resource Description Framework (RDF); Available from: http://www.w3.org/RDF/

24. SKOS Editor, University of Manchester, 2008; Available from: http://protegewiki.stanford.edu/wiki/SKOS Editor 